\title{
Comparative analysis of forecasting portfolio returns using Soft Computing technologies
}

\author{
A. Rubio, J.D. Bermúdez, E. Vercher \\ Department of Statistics and Operations Research, University of Valencia, Spain.
}

\begin{abstract}
We propose using fuzzy time series (FTS) to forecast the future performance of returns on portfolios. We model the portfolio selection problem by means of possibilistic moments, and approximate the uncertainty of the return on a given portfolio by trapezoidal fuzzy numbers.

Some modifications into the classical models of FTS, based on weighted operators, allow us generating trapezoidal numbers as forecasters of the performance of portfolio returns. We incorporate our proposals into classical FTS methods and analyze their effectiveness with respect to FTS models with a possibilistic interval-valued mean approach, using historical returns on assets from the Spanish stock market.
\end{abstract}

Keywords: Fuzzy Time Series, Possibilistic moments, Portfolio Selection, Forecasting

\section{Introduction}

Since the introduction of portfolio selection problem by Markowitz in 1952, a lot of different models, approaches and procedures have been developed in order to suitable managing the uncertain behavior of markets, the experience or beliefs of experts and investors wishes. The origin of modern portfolio theory is the mean-variance probabilistic model [1]; where the trade-off between return and risk of the investment is the goal for the decision maker, using probability theory and mathematical optimization as the main tools for achieving an efficient portfolio. The process of selecting an optimal portfolio may be divided into two stages: the first stage starts with observation of the behavior of returns on assets, incorporating the experience and knowledge of experts, and finalizes with beliefs about the future performance of available portfolios (throughout the individual performance of securities and the jointly performance of the portfolio). The second stage concerns the procedures designed for finding and select the portfolio which is optimal with respect to one single or multiple objectives, fulfilling the requirements imposed by the investor.

We can find several and suitable Soft Computing approaches to the portfolio selection problem in the two stages of the selecting process: in the modeling of beliefs and the incorporation of imprecise knowledge, for the approximation of uncertain performance of future returns on assets and portfolios and in the development of optimization procedures, mainly through fuzzy mathematical programming techniques and evolutionary algorithms $[2,3,4,5]$.

Fuzzy modeling of time series has been proposed by Song and Chissom $[6,7,8]$, introducing timevariant and time-invariant models to forecast the enrollment of students at Alabama University. The major points in their modeling approach is related to the partitioning of the universe of discourse, the establishment of fuzzy relationship from the fuzzy time series and the process of forecasting and defuzzification of the outputs [9]. Concerning the partitioning of the universe of discourse, different proposals to determine suitable intervals have been considered, in such a way that the determination of effective length of intervals affects forecasting results in fuzzy time series $[10,11]$. To state appropriate fuzzy relationships is also critical in FTS, a very interesting approach is proposed in [12], where the weighted fuzzy time series model considered recurrent fuzzy relationships for the assignation of weights of each individual relationship, which outperform the forecasting. However, with respect to forecasting proposals most authors follows the scheme originally suggested by Song and Chissom. In our context, fuzzy approaches for solving time series problems have been applied in stock indices forecasting and for modeling business cycles (see, for instance, $[12,13,14,15,16])$. While forecasting returns on a given portfolio has not been treated in previous researches.

Usually the proposed forecasts by current fuzzy time series models are looking for a single-point value just like the output of classical time series methods. But, for ranking portfolios we need to know not only their expected return but a measure of the investment risk. In previous works, we have deal with LR-type fuzzy numbers as useful approximation of the uncertainty of returns on individual assets or a given portfolio, for which possibilistic moments allow us to calculate the value, the ambiguity and some significantly properties of the fuzzy number $[17,5,18]$. In the present research our main goal is designing a fuzzy time series modeling approach for providing trapezoidal fuzzy numbers as forecasts of future performance of return on a given portfolio, using the historical data set of portfolio returns. In our opinion this information will 
be useful to the decision analyst once it has been incorporated into the fuzzy mathematical portfolio selection models, which usually approximates the future performance of returns without considering their temporality. Additionally, to work with trapezoidal fuzzy numbers will allow us to analyze both expected return and risk of a particular investment, which is an appropriate financial approach to the fuzzy portfolio selection problem.

The remaining content of this paper is organized as follows. In Section 2, we review the fuzzy portfolio selection problem when possibility theory have been used to measure the uncertainty of future portfolio returns. Section 3 provides a review of fuzzy time series definitions and methods, including new proposals for improving the fuzzy forecasting of time series. Finally, in Section 4 we analyze the comparative performance of classical fuzzy time series vs the proposed modifications using a historical data set from the returns on assets of the IBEX35 (the stock index of the Spanish stock market). Conclusions are presented in Section 5.

\section{Possibilistic portfolio selection models}

The main approaches to the portfolio optimization problem have the objective of maximizing the return on the investment with the counterpart of minimizing the risk of the inversion, being risk and return quantitative variables that held certain correlation between them. Additionally, it is well accepted that the future performance of investment is uncertain, then fuzzy set theory have been widely used: for describing the imperfect knowledge of asset returns and other imprecise data affecting financial stock markets, for modeling the selection problem by means of fuzzy goals and constraints and for solving the corresponding optimization problems using Soft Computing techniques. Our present work deals with risky portfolios, whose uncertainty on their expected returns is modeled by using trapezoidal fuzzy numbers.

The vector $X=\left(x_{1}, \ldots, x_{n}\right)$ represents the allocation of the total budget over different risky assets, $x_{i}$ being the fraction invested in asset $i, i=1, \ldots, n$. Let us represent the uncertainty regarding the return on a given portfolio, $P_{X}$, using a trapezoidal fuzzy number, $\tilde{T}_{X}=\left(t_{l}, t_{u}, \alpha, \beta\right)$. The core of the trapezoidal fuzzy number $\tilde{T}_{X}$ is the interval $\left[t_{l}, t_{u}\right]$ and the quantities $\alpha$ and $\beta$ are their leftand right-spreads, respectively. Additionally, we estimate the expected return on and the risk of the investment by means of possibilistic interval-valued means and fuzzy downside-risk of $\tilde{T}_{X}$, respectively $[19,20,21,17]$. Note that we directly approximate the core and spreads of the trapezoidal fuzzy return on a given portfolio, instead of approximate the returns on individual assets as trapezoidal fuzzy numbers. This alternative approach to quantify uncertainty of the portfolio returns, allows us to incor- porate a certain measure of the relationship among the returns on the assets with respect to their contemporary behavior [22].

In the specific case of trapezoidal fuzzy numbers, it can easily be checked that following the definition of Dubois and Prade [19], the interval-valued mean of $\tilde{T}_{X}$ is the following interval:

$$
I_{m}\left(\tilde{T}_{X}\right)=\left[t_{l}-\alpha / 2, t_{u}+\beta / 2\right] .
$$

This interval corresponds to the half-cut of $\tilde{T}_{X}$, and it is also the $f$-weighted interval-valued possibilistic mean introduced in [21] for the weighted function $f(\gamma)=1$. Concerning the crisp modeling of the expected return, we will use the middle point of the corresponding interval-valued mean as the mean value of $\tilde{T}_{X}$ :

$$
E\left(\tilde{T}_{X}\right)=\left(t_{l}+t_{u}\right) / 2+(\beta-\alpha) / 4
$$

The $f$-weighted interval-valued possibilistic mean value of a fuzzy number [21] can be considered as a generalization of both the interval-valued mean [19] and the interval-valued possibilistic mean [20], using the weighting functions $f(\gamma)=1$ and $f(\gamma)=$ $2 \gamma$, respectively. The authors also noted that their definition of the f-weighted possibilistic mean value coincides with the value of a fuzzy number (with respect to the reducing function $0.5 f$ ) introduced by Delgado et al. [23].

The downside risk of $P_{X}$ measures the failure to achieve the interval-valued mean by using the mean absolute semi-deviation, which penalizes only the negative deviations of the expected return [17]. For a more detailed explanation of using these fuzzy measures for the possibilistic portfolio selection problem readers are referred to [17], where some relationships between different interval-valued definitions are also presented.

\section{Fuzzy Time Series}

The classical time series forecasting methods can not deal with problems in which the values of time series are linguistic terms represented by fuzzy sets. So, Song and Chissom [6] developed fuzzy time series to overcome this drawback. Let us briefly review some definitions of FTS introduced in $[7,8,9,24]$.

Definition 1. Let $U=\left\{u_{1}, \ldots, u_{m}\right\}$ the universe of discourse. A fuzzy set $A$ of the universe of discourse $U$ can be defined as follows:

$A=f_{A}\left(u_{1}\right) / u_{1}+f_{A}\left(u_{2}\right) / u_{2}+\ldots+f_{A}\left(u_{m}\right) / u_{m}$

where $u_{i}$ is an element of $U, f_{A}$ is the membership function of $A, f_{A}: U \rightarrow[0,1]$ denotes the membership degree of $u_{i}$ in the fuzzy set $A$ for $i=1, \ldots, m$.

Definition 2. Let $Y(t)$ a subset of real numbers, being the universe of discourse in which fuzzy sets $f_{i}(t)$ are defined. Let $F(t)$ be a collection of $f_{i}(t)$, 
$i=1,2, \ldots$, then $F(t)$ is called a fuzzy time series on $Y(t), t=\ldots 0,1,2, \ldots$.

Definition 3. Let $F(t-1)=A_{i}$ and $F(t)=A_{j}$. The relationship between two consecutive observations, $F(t-1)$ and $F(t)$, referred to as a fuzzy logical relationship, is denoted by $F(t-1) \rightarrow F(t)$ or by $A_{i} \rightarrow A_{j}$, where $A_{i}$ is called the left-hand side and $A_{j}$ the right-hand side of the fuzzy logical relationship.

Fuzzy logical relationships with the same fuzzy set located in the left-hand side of the relationships can be further grouped into a fuzzy logical relationship group [24].

Definition 4. Suppose that there are fuzzy logical relationships in such a way that $A_{i} \rightarrow A_{j 1}$, $A_{i} \rightarrow A_{j 2}$, and so on, they can be grouped into a fuzzy logical relationship group $A_{i} \rightarrow A_{j 1}, A_{j 2}, \ldots$.

\subsection{Fuzzy time series forecasting methods}

Since the publication of the works of Song and Chissom $[6,7,8]$ many methods have been proposed for forecasting fuzzy time series. The basic steps of the forecasting process in fuzzy time series are the following:

1. Partitioning the universe of discourse. Which includes the definition of the universe of discourse $U$ and the number and length of the intervals.

2. Defining the fuzzy sets on $U$ and fuzzifying time series with the use of these fuzzy sets. Fuzzification step using Def. 1.

3. Establishing fuzzy logical relationships from the fuzzy time series. These relationships are based on the historical data and permit to derive fuzzy logical relationship groups.

4. Calculating the forecast values. Locate the linguistic value of the last observed data point (left-hand side) and use the midpoint of each linguistic value at the right-hand side of its fuzzy logical relationship for calculate the forecast: the arithmetic mean of all these midpoints.

Let us introduce Chen proposals [9] and the modifications suggested by Huarng $[10,24]$ in order to establish the partitioning of $U$ in the first step. In the Chen method, the universe of discourse $U$ is defined from min and max data values: $D_{\min }$ and $D_{\text {max }}$, respectively (obtained among all historical data $\left.D_{i}, i=1, \ldots, N\right)$; in such a way that $U=$ $\left[D_{\min }-d_{1}, D_{\max }+d_{2}\right]$, being $d_{1}$ and $d_{2}$ two small numbers. Once the length of the universe of discourse is determined, $U$ can be partitioned into several equal length intervals (usually seven intervals). Huarng [10] observed that the length of the interval affects the accuracy of the forecasting results, then he proposed two methods for determining the unequal length of intervals: Distribution-based length method (named Huarng-1) and Averagebased length method (named Huarng-2).
1. Distribution-based length method.

(a) Calculate all the absolute differences between $D_{i+1}$ and $D_{i}$ from $i=1, \ldots, N-1$, as the first differences and compute the average of the first differences.

(b) Determine the base for the length of intervals by following an heuristic rule, taking into account the average of the first differences.

(c) According to the assigned base, choose as the length of intervals the largest length that is smaller than at least half of the first differences.

2. Average-based length method.

(a) Since the average of the first differences between $D_{i+1}$ and $D_{i}$ may not necessarily fulfill the heuristic rule, the averagedbased length is set to taking one-half of the average as the length.

(b) According to the assigned base, round the length as the appropriate $l$ and compute the number on intervals, $m$.

So, following the Huarng proposals there are $m$ intervals and $m$ fuzzy sets $\left(u_{i}\right.$ and $A_{i}$, resp.), and each observation in the training data set has a related fuzzy set (linguistic value). If the observed value is located in the range of $u_{i 0}$, then it belongs to fuzzy set with a greater membership value (usually $\left.A_{i 0}\right)$. The fuzzy sets, $A_{i}$ for $i=1, \ldots, m$, for the universe of discourse $U$ are defined by using Eq. (3). For all fuzzified data we state the fuzzy logical relationships using Defs. 3 and 4.

Note that the main differences between Chen and Huarng FTS methods are in the rules for the partitioning and determining the length of intervals, since the authors also use the same definitions in the intermediate steps and the same rules for calculating the crisp forecasts. Other authors introduce alternative procedures to provide a partition of the universe of discourse into intervals of unequal length $[11,15]$; their proposals for Step 1 are based on fuzzy clustering techniques and information granules and they can improve the crisp forecast accuracy.

Alternatively, Yu [12] modifies Step 3, establishing fuzzy logical relationships which incorporate chronologically-determined weights in order to reflect recurrence; Yu FTS method also uses its particular weighted average in the defuzzification process. Following this research line, Cheng et al [25] propose weighting the relationships among the different fuzzy sets (based on frequency), and Lee et al [26] present other modified weighted versions of Yu FTS method.

On the other hand, Liu [27] FTS forecasting method also maintains the partitioning of the universe of discourse suggested by Huarng (the average-based length method), but calculates the forecasted outputs as trapezoidal fuzzy numbers, using the endpoints of the intervals $u_{i}$. 
In this paper we propose several modification of the above procedures. Firstly, in Step 2 the algorithm defines the membership function of every linguistic value $A_{i}$ as a trapezoidal fuzzy number; secondly, it builds the fuzzy forecast using the following heuristic rules:

1. If the fuzzy logical relationship group of $A_{j}$ is empty, $A_{j} \rightarrow \emptyset$, the procedure builds a trapezoidal fuzzy number with all values belonging to $A_{j}$.

2. If the fuzzy logical relationship group of $A_{j}$ goes to $A_{k}$, the procedure builds a trapezoidal fuzzy number with all values that belongs to $A_{k}$

3. If the fuzzy logical relationship group of $A_{j}$ goes to many $A_{k 1}, A_{k 2}, \ldots, A_{k r}$, the procedure builds a trapezoidal fuzzy number with all values that belongs to $A_{k 1}, A_{k 2}, \ldots, A_{k r}$. Taking into account the frequency of each one-to-one relationship using the observed data set.

Finally, in order to compare this weighted fuzzy approach with the classical FTS forecasting proposals, the procedure calculates the weighted average of the possibilistic mean values of the trapezoidal fuzzy numbers representing the right-hand side linguistic values as a pointwise forecast. We include these modifications of Steps 2 and 4 into the methods of Chen and Huarng, which are denoted with the surname -weighted, in order to analyze if they provide any improvement in the forecasting accuracy.

\section{A simulation experiment}

In order to check the predictive accuracy of the forecast methods described in the previous section we run a simulation experiment. We randomly select portfolios built by considering the returns on $n=23$ assets included in the IBEX35, the Spanish stock market index. We introduce some usual constraints for selecting feasible portfolios. In order to obtain more diversification in the investment we impose an upper bound $u$ to the proportion of the investment on each asset, namely $u=0.3$, so we are demanding to each portfolio $X=\left(x_{1}, \ldots, x_{23}\right)$ that $x_{i} \leq 0.3 \forall i$, and we also introduce a cardinality constraint for the purpose of control, namely $K=7$, being $K$ the number of assets included in the portfolio with positive proportion of investment, portfolio size. Then, Our set of feasible portfolios is the subset of the simplex of the 23-dimensional real space subject to the conditions given by the above constraints and the condition of total investment: $\sum_{i=1}^{23} x_{i}=1$.

Each portfolio $X$ in the simulation experiment is obtained by the following procedure:

Step 1. A realization of the uniform distribution on the simplex of dimension $K$ is computed using the algorithm proposed in [5]. This step is repeated till every component of this $K$ dimensional vector is smaller than the upper bound. The output is a vector $p=\left(p_{1}, \ldots, p_{K}\right)$ with components belonging to the open interval $(0, u)$ and fulfilling that $\sum_{i=1}^{K} p_{i}=1$.

Step 2. $K$ assets are selected by simple sampling from the $n$ available.

Step 3. Finally, the portfolio $X$ is obtained assigning the $p$ values to the $K$ selected assets and the zero value to the other assets.

This procedure ensures that each portfolio has been uniformly selected from the feasible set.

The training data set consists of 151 weekly returns on those assets observed throughout the time window January 2011 to December 2013, being the return on asset $i$ at week $t$ defined as follows:

$$
r_{t i}=100\left(P_{t i}-P_{(t-1) i}\right) / P_{t i}
$$

for $t=2, \ldots, 152$ and $i=1, \ldots, 23$, where $P_{t i}$ is the closed price of asset $i$ at Wednesday of week $t$. For every selected portfolio $X$ we evaluate its weekly return on at each Wednesday as follows:

$$
r_{t}(X)=\sum_{i=1}^{23} r_{t i} x_{i} .
$$

We are going to used the first 150 returns on portfolio $P_{X}$ as training data set and will use them to predict the next return.

\subsection{Forecasting the return on a single portfolio}

As an example of the forecast analysis of those portfolios we are going to summarize the forecast of Portfolio \#1, the first simulated one, using Chen and Chen-weighted methods. Figure 1 shows the time series of returns on Portfolio \#1.

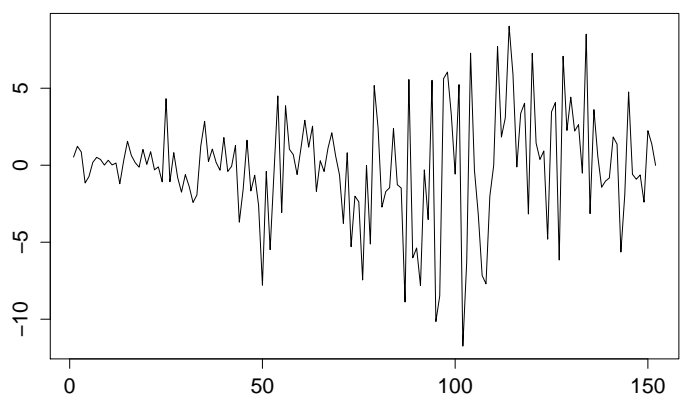

Figure 1: Trace of the time series of returns on Portfolio \#1.

In order to apply Chen's forecasting method [9], the universe of discourse is partitioned into seven intervals, all of equal length and with the first one beginning at -14.05 ; those intervals allows us to define the seven fuzzy sets which are the linguistic 


\begin{tabular}{crlr}
\hline Lin. value & Midpoint & Rel. Group & Forecast \\
\hline$A_{1}$ & -12.2 & $A_{3}$ & -5.00 \\
$A_{2}$ & -8.6 & $A_{2}, A_{4}, A_{6}$ & -1.36 \\
$A_{3}$ & -5.0 & $A_{2}, \ldots, A_{6}$ & -1.36 \\
$A_{4}$ & -1.4 & $A_{2}, \ldots, A_{7}$ & 0.45 \\
$A_{5}$ & 2.3 & $A_{3}, \ldots, A_{7}$ & 2.27 \\
$A_{6}$ & 5.9 & $A_{1}, \ldots, A_{6}$ & -3.17 \\
$A_{7}$ & 9.5 & $A_{4}, \ldots, A_{6}$ & 2.27 \\
\hline
\end{tabular}

Table 1: Intermediate results in Chen forecasting method.

values of the linguistic variable 'return'. Those fuzzy sets and the midpoint of their related intervals appear in the first two columns of Table 1. The third column shows the fuzzy logical relationship groups while the last column shows the crisp point forecast, which is the arithmetic mean of the midpoints of the linguistic values belonging to the respective fuzzy logical relationship group.

Let us denote by $r_{(t, \# m)}$ the return on Portfolio $\# \mathrm{~m}$ observed at time $\mathrm{t}$. Then, the last observed value in the training set of this time series is $r_{(150,1)}=1.39$, which belongs to the fifth interval, so its fuzzyfied linguistic value is $A_{5}$, and applying results in Table 1 the crisp point forecast is $\widehat{r}_{1(151,1)}=2.27$. Since the actual observed value was $r_{(151,1)}=-0.01$, the prediction error is $e_{1,1}=\widehat{r}_{1(151,1)}-r_{(151,1)}=2.28$.

Alternatively, we realize that the elements in each fuzzy logical relationship group appears with very different frequencies; for example the relation $A_{5} \rightarrow A_{3}$ was observed only five times while the relation $A_{5} \rightarrow A_{4}$ has a frequency of 20 times along the training set. We use this information in the so-called Chen-weighted method, which takes into account those frequencies, and provides as pointwise fuzzy forecast a weighted average of the linguistic values of the linguistic variable 'return', with weights proportional to the relative frequencies of the elements in the corresponding fuzzy logical relationship group. In addition, following [27] we define the membership function of the seven linguistic values with trapezoidal numbers.

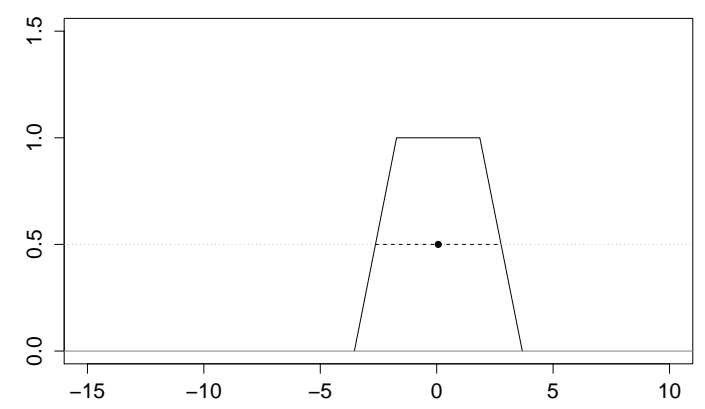

Figure 2: Membership function of the fuzzy forecast for the the time series used in the example. The midpoint of the $0.5 \gamma$-cut is the crisp forecast.
Figure 2 shows the trapezoidal number representing the membership function of the fuzzy forecast obtained for the time series of Portfolio \#1. This number is a weighted average of the trapezoidal numbers representing the seven linguistic values that compose the universe of discourse; the weights are given by the vector $(0.0,0.0,0.11,0.44,0.40,0.02,0.02)$ whose components are the relative frequencies of the fuzzy logical relationship of $A_{5}$ (the linguistic value of the last observed value in the training set $\left.r_{(150,1)}=1.39\right)$ with the other linguistic values into its relationship group. The current crisp point forecast is the possibilistic valued-mean of this trapezoidal fuzzy number, which is the midpoint of its $\gamma$-cut, with $\gamma=0.5$ (Eq. 2).

The new crisp forecast is represented in Figure 2 with a bold point and takes the following value: $\widehat{r_{2}(151,1)}=0.07$. The prediction error obtained with this proposal applied to the time series of returns of Portfolio \#1 is $e_{1,2}=\widehat{r}_{2(151,1)}-r_{(151,1)}=0.08$, sensibly smaller than the forecasting error obtained using Chen forecasting method.

\subsection{Results of the simulation experiment}

In order to compare the performance of the above forecasting proposal, we obtain a set of $N=1000$ randomly simulated portfolios and forecast all of them using the six forecasting methods described in the previous section. For each portfolio $X_{i}$ and every method $M_{j}$, we compute the forecasting error $e_{i, j}=\widehat{r}_{(151, i)}-r_{(151, i)}$, being $\widehat{r}_{(151, i)}$ the forecast and $r_{(151, i)}$ the actually observed return on that portfolio. Figure 3 shows the boxplot description of all those forecasting errors.

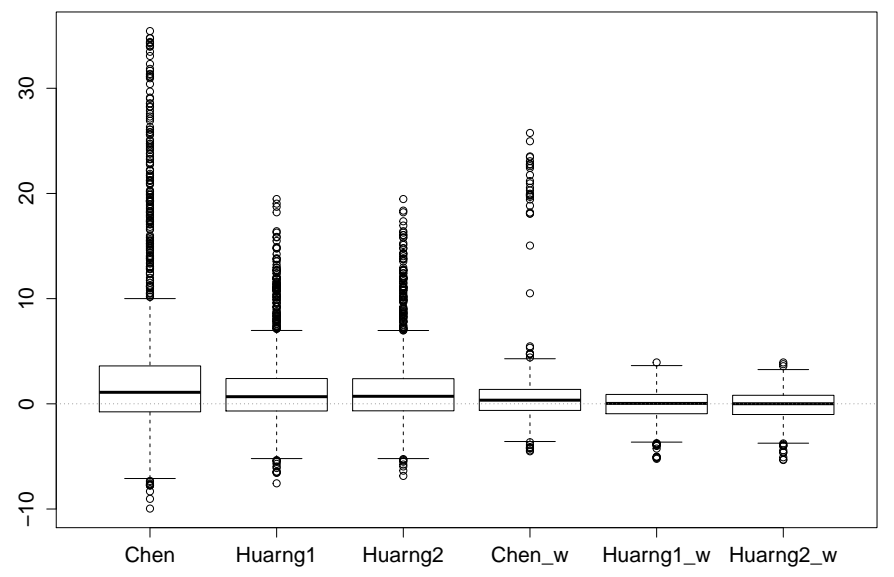

Figure 3: Boxplot description of the forecasting errors obtained in the simulation experiment.

For the results of the simulation experiment, we compute three accuracy measures: the arithmetic mean of the observed errors, Bias $=\sum_{i=1}^{N} e_{i, j} / N$; the mean of their absolute values, MAD = $\sum_{i=1}^{N}\left|e_{i, j}\right| / N$, and the mean of the squared errors, 


\begin{tabular}{lrrr}
\hline Method & Bias & MAD & MSE \\
\hline Chen & 3.65 & 5.13 & 81.33 \\
Huarng-1 & 1.55 & 2.74 & 18.56 \\
Huarng-2 & 1.58 & 2.68 & 18.26 \\
Chen-weighted & 0.77 & 1.70 & 13.48 \\
Huarng-1-weighted & -0.10 & 1.12 & 2.03 \\
Huarng-2-weighted & -0.13 & 1.13 & 2.07 \\
\hline
\end{tabular}

Table 2: Forecasting accuracy methods obtained in the simulated experiment.

MSE $=\sum_{i=1}^{N} e_{i, j}^{2} / N$. The first measure is an estimate of the bias of the method, ideally it should be zero. The second and third measures are the mean absolute error and the mean squared error, respectively; both usual forecast accuracy measures. The numerical results obtained are shown in Table 2 .

Note that the first four methods produce forecast errors that are very asymmetrical and more dispersed than the last two methods (Huarngweighted), as Figure 3 explicitly shown. That behavior is also reflected in Table 2: the mean squared errors of the two last methods are significatively smaller than the MSE provided by the other methods.

\section{Conclusion}

We consider some classical Fuzzy Time Series methods in which we incorporate several modifications in the process of forecasting and defuzzification of the outputs, based on weighted operators. Our approach allows providing trapezoidal fuzzy numbers which are better forecasts of future performance of the return on a given portfolio, for which we forecast return and risk. We have developed a simulation experiment using a data set from the Spanish stock market in order to analyze the effect of the proposed modifications. In summary:

1. Every of the analyzed methods has been improved using weighted means: the bias is closer to zero and the MAD and MSE diminish substantially.

2. We did not found practical differences between the results obtained with the two Huarng methods (Distribution-based length method and Average-based length method), neither using arithmetic means nor using weighted means.

3. In this simulation study, Chen method, with intervals of the same length, has had the worst performance concerning the forecasting errors.

Some future studies could focus on applying our approach on FTS methods using intervals of unequal lengths.

\section{Acknowledgment}

The authors would like to thank the anonymous reviewers for their valuable comments and sugges- tions, which helped us to improve an earlier version of this paper.

This research was partially supported by the Ministerio de Economia y Competitividad, Spain (Research Project MTM2014-56233-P).

\section{References}

[1] H.M. Markowitz, Portfolio selection, Journal of Finance, 7:77-91, 1952.

[2] M. Inuiguchi and J. Ramík, Possibilistic linear programming: a brief review of fuzzy mathematical programming and a comparison with stochastic programming in portfolio selection problem, Fuzzy Set Systems, 111:3-28, 2000.

[3] T. León, V. Liern and E. Vercher, Viability of infeasible portfolio selection problems: a fuzzy approach, European Journal of Operational Research, 139:178-189, 2002.

[4] J.L. Verdegay, R.R. Yager and P.P. Bonissone, On heuristics as a fundamental constituent of soft computing, Fuzzy Sets and Systems, 159(7):846-855, 2008.

[5] J.D. Bermúdez, J.V. Segura and E. Vercher, A multi-objective genetic algorithm for cardinality constrained fuzzy portfolio selection, Fuzzy Sets and Systems, 188:16-26, 2012.

[6] Q. Song and B.S. Chissom, Forecasting enrollments with fuzzy time series - part I, Fuzzy Sets and Systems, 54:1-9, 1993.

[7] Q. Song and B.S. Chissom, Fuzzy time series and its models, Fuzzy Sets and Systems, 54:269277, 1993.

[8] Q. Song and B.S. Chissom, Forecasting enrollments with fuzzy time series - part II, Fuzzy Sets and Systems, 62:1-8, 1994.

[9] S.M. Chen, Forecasting enrollments based on fuzzy time series, Fuzzy Sets and Systems, 81:311-319, 1996.

[10] K.H. Huarng, Effective lengths of intervals to improve forecasting in fuzzy time series, Fuzzy sets and Systems, 123:387-394, 2001.

[11] L. Wang, X. Liu and W. Pedrycz, Effective intervals determined by information granules to improve forecasting in fuzzy time series, Expert Systems with Applications, 40:5673-5679, 2013.

[12] H-K. Yu, Weighted fuzzy time series models for TAIEX forecasting, Physica A, 349:609-624, 2005.

[13] K.H. Huarng and H-K. Yu, A Type 2 fuzzy time series model for stock index forecasting, Physica A, 353:445-462, 2005.

[14] K.C. Hung and K.P. Lin, Long-term bussines cycle forecasting through a potential intuitionistic fuzzy least-squares support vector regression approach, Information Sciences, 224:37-48, 2013.

[15] L. Wang, X. Liu, W. Pedrycz and Y. Shao, Determination of temporal information granules to 
improve forecasting in fuzzy time series, Expert Systems with Applications, 41:3134-3142, 2014.

[16] Y-S. Chen, C-H. Cheng and W-L. Tsai, Moddeling fiiting-function-based fuzzy time series patterns for evolving stock index forecasting, Appl Intell., DOI 10.1007/s10849-014-0520-6.

[17] E. Vercher, J.D. Bermúdez and J.V. Segura, Fuzzy portfolio optimization under downside risk measures, Fuzzy Sets and Systems, 158:769782, 2007.

[18] E. Vercher, J.D. Bermúdez, A possibilistic Mean-Downside Risk-Skewness model for efficient portfolio selection IEEE Transactions on Fuzzy Systems, 21(3):585-595, 2013.

[19] D. Dubois and H. Prade, The mean value of a fuzzy number, Fuzzy Sets and Systems, 24:279300, 1987.

[20] C. Carlsson and R. Fullér, On possibilistic mean value and variance of fuzzy numbers, Fuzzy Sets and Systems, 122:315-326, 2001.

[21] R. Fullér and P. Majlender, On weighted possibilistic mean and variance of fuzzy numbers, Fuzzy Sets and Systems, 136:363-374, 2003.

[22] J.D. Bermúdez, J.V. Segura and E. Vercher, A fuzzy ranking strategy for portfolio selection applied to the Spanish stock market, Fuzzy Systems Conference, 2007, FuzzyIEEE2007 IEEE International, 1-4, doi: 10.1109/FUZZY.2007.4295466.

[23] M. Delgado, M.A. Vila and W. Voxman, On a canonical representation of fuzzy numbers, Fuzzy Sets and Systems, 93:125-135, 1998.

[24] K.H. Huarng, Heuristic models of fuzzy time series for forecasting, Fuzzy sets and systems, 123:369-386, 2001.

[25] C.H. Cheng, T.L. Chen, H.J. Teoh and C.H. Chiang, Fuzzy time series based on adaptative expectation model for TAIEX forecasting, Expert Systems with Applications, 34(2): 11261132, 2008.

[26] M.H. Lee, R. Effendi and Z. Ismail, Modified weighted for enrollment forecasting based on fuzzy time series, MATHEMATIKA, 25(1):67$78,2009$.

[27] H-T. Liu, An improved fuzzy time series fprecasting method using trapezoidal fuzzy numbers, Fuzzy Optimization and Decision Making, 6:63-80, 2007. 